\title{
Exercise-induced hyperthermia syndrome (canine stress syndrome) in four related male English springer spaniels
}

This article was published in the following Dove Press journal:

Veterinary Medicine: Research and Reports

I September 2017

Number of times this article has been viewed

\section{Elizabeth Thrift ${ }^{1}$ \\ Justin A Wimpole 2 \\ Georgina Child ${ }^{2}$ \\ Narelle Brown' \\ Barbara Gandolfi ${ }^{3}$ \\ Richard Malik ${ }^{4}$}

'Animal Referral Hospital, ${ }^{2}$ Small Animal Specialist Hospital, Sydney, NSW, Australia; ${ }^{3}$ Veterinary Medicine and Surgery, College of Veterinary Medicine, University of Missouri, Columbia, MO, USA; ${ }^{4}$ Centre for Veterinary Education, University of Sydney, Sydney, NSW, Australia
Correspondence: Elizabeth Thrift Animal Referral Hospital, 250 Parramatta Road, Homebush West, Sydney, NSW

2|40, Australia

Tel +6I 40999336 I

Email e.thrift@arhvets.com
Objective: This retrospective study describes the signalment, clinical presentation, diagnostic findings, and mode of inheritance in four young male English springer spaniel dogs with presumptive canine stress syndrome.

Materials and methods: Appropriate cases were located through medical searches of medical records of two large private referral centers. Inclusion criteria comprised of English springer spaniel dogs with tachypnea and hyperthermia that subsequently developed weakness or collapse, with or without signs of hemorrhage, soon after a period of mild-to-moderate exercise. The pedigrees of the four affected dogs, as well as eleven related English springer spaniels, were then analyzed to determine a presumptive mode of genetic inheritance.

Results: Four dogs met the inclusion criteria. All four were male, suggesting the possibility of a recessive sex-linked heritable disorder. Pedigree analysis suggests that more dogs may be potentially affected, although these dogs may have never had the concurrent triggering drug/ activity/event to precipitate the clinical syndrome. There was complete resolution of clinical signs in three of the four dogs with aggressive symptomatic and supportive therapy, with one dog dying during treatment.

Conclusion: Dogs with canine stress syndrome have the potential for rapid recovery if treated aggressively and the complications of the disease (eg, coagulopathy) are anticipated. All four dogs were male, suggesting the possibility of a recessive sex-linked mode of inheritance. Further genetic analyses should be strongly considered by those involved with the English springer spaniel breed, either with a genome-wide association study using canine single-nucleotide polymorphism arrays or whole-genome sequencing of affected and closely related dogs.

Keywords: malignant hyperthermia, canine stress syndrome, coagulopathy, dog, English springer spaniel

\section{Introduction}

Malignant hyperthermia (MH) is a clinical syndrome that has been reported in a variety of species, most notably humans, pigs, and dogs. ${ }^{1,2}$ When fully developed, it is characterized by muscle rigidity, hyperthermia (often rapid in onset), tachypnea, metabolic acidosis, hypercarbia, and cardiac arrhythmias. ${ }^{3}$ More subtle or abortive presentations of canine $\mathrm{MH}$ have also been reported. Furthermore, several classic signs of porcine and human $\mathrm{MH}$ are typically lacking in canine $\mathrm{MH}$ patients. For example, canine $\mathrm{MH}$ is not typically characterized by lactic acidosis or the early onset of muscle rigidity.

The term "canine stress syndrome" (CSS) has been borrowed from pigs (viz porcine stress syndrome) and echoed in subsequent publications to describe such cases, but the entity remains poorly defined. ${ }^{4,5}$ It is often used in relation to both true MH cases 
and exercise-induced hyperthermia syndrome (EIHS) which shares some similarities with MH. EIHS/CSS is generally self-limiting, responding to aggressive supportive therapy, without the requirement for specific therapy with dantrolene. EIHS has been identified in many breeds, including a male English springer spaniel (ESS) and a greyhound. ${ }^{6,7}$

EIHS/CSS is apparently precipitated by moderate-tosevere exercise in genetically susceptible dogs, although it is plausible additional triggers need to be present concurrently. The syndrome initially consists of a variable combination of hyperthermia, muscle cramping, tachypnea, and eventually collapse, with or without dyspnea. Some dogs develop seizures as part of the syndrome. Clinicopathologic abnormalities include hemoconcentration, hyperlactatemia, respiratory alkalosis, and increased muscle-enzyme activities in serum. ${ }^{5}$ Later, consumptive coagulopathy can develop, in addition to a variety of other life-threatening sequelae.

The following case series describes four suspected cases of EIHS/CSS and subsequent investigations suggesting a possible hereditary predisposition. All four affected individuals were entire male ESSs that were 2-3 years of age at the time of presentation. All were closely related. This is the first case series of EIHS cases, and our findings echo the first case recorded by Rand and O'Brien, also in an entire male ESS. ${ }^{6}$

\section{Materials and methods}

Medical records of the Small Animal Specialist Hospital and the Animal Referral Hospital between 2012 and 2014 were searched using the respective practice-management software utilizing the keywords "exercise-induced", "hyperthermia", and "heat stroke". Four ESSs with signs consistent with EIHS and collapse were located. Data retrieved from the records included signalment, historical observations, clinical signs, physical findings, laboratory-test results, imaging findings, treatment protocols, and outcome (including whether the patient survived or not and whether disease recurred subsequently). The pedigrees of all affected dogs were retrievable. Our preliminary findings were presented at College Science Week in July 2015, the annual meeting of the Australian and New Zealand College of Veterinary Scientists, in an attempt to obtain data from other similarly affected dogs. Unfortunately, no further cases could be recruited into this study.

\section{Results}

\section{Signalment and seasonal occurrence}

All four patients were entire male ESSs. All were used by the police as "sniffer dogs" for the detection of illicit drugs, flares, explosives, and so forth. The dogs were part of the same police-dog unit, although they were housed in different homes, with their respective handlers. One dog presented at 23 months of age, one at 26 months of age, and two at 32 months (although one of these dogs had a previous episode of possible collapse at 22 months of age, but did not require veterinary attention at the time). Two of the four dogs presented in spring, one presented in summer, and the final dog presented in autumn (Table 1).

\section{Historical findings}

All dogs presented with a history of collapse or weakness after mild-to-moderate exercise. Three of the four dogs were tachypneic, which did not improve with rest. Three of the four dogs became weak, which progressed to complete collapse. Two dogs had clinical evidence of coagulopathy with spontaneous bleeding. Two of the four dogs subsequently vomited. Three of the four dogs presented to a veterinarian within 24 hours of an exercise-induced illness developing. The remaining dog presented after more than 24 hours but less than 48 hours after the antecedent event (Table 1).

\section{Physical and neurological examination findings on presentation}

Hyperthermia (rectal temperature, $>39.2^{\circ} \mathrm{C}$ ) was present in three of the four dogs (Table 1). The highest recorded rectal temperature for any case was $41^{\circ} \mathrm{C}$. All four dogs were tachypneic. Three were tachycardiac. Two were nonambulatory and obtunded. One of the four dogs was ambulatory, but "ataxic" (or weak) in both pelvic limbs. Two dogs had evidence of a

Table I Summary of signalment in four male entire English springer spaniels with exercise-induced hyperthermia (canine stress syndrome)

\begin{tabular}{|c|c|c|c|c|}
\hline Clinical features & Case I & Case 2 & Case 3 & Case 4 \\
\hline $\begin{array}{l}\text { Age at presentation } \\
\text { (months) }\end{array}$ & 26 & 23 & 32 & 32 \\
\hline $\begin{array}{l}\text { Veterinary } \\
\text { intervention }\end{array}$ & $\begin{array}{l}\text { Same } \\
\text { day }\end{array}$ & $\sim 36$ hours & Same day & $<6$ hours \\
\hline Season & Spring & Spring & Autumn & Summer \\
\hline Tachycardia & Yes & Yes & Unknown & Yes \\
\hline Tachypnea & Yes & Yes & Yes & Yes \\
\hline Ambulatory & No & Yes & $\begin{array}{l}\text { Yes, but } \\
\text { "ataxic" in } \\
\text { all limbs }\end{array}$ & No \\
\hline Hemorrhage & Yes & $\begin{array}{l}\text { Yes (footpads, } \\
\text { ecchymoses, } \\
\text { and melena) }\end{array}$ & No & No \\
\hline $\begin{array}{l}\text { Rectal body } \\
\text { temperature }\left({ }^{\circ} \mathrm{C}\right)\end{array}$ & 40.1 & 38.8 & 39.3 & 39.9 \\
\hline Outcome & Survived & Survived & Died & Survived \\
\hline
\end{tabular}


coagulopathy, one with hemorrhage from the prepuce and carpal/tarsal pads of three paws, while the other bled from both forepaws. Other abnormalities included a generalized motor seizure (one dog), muscle fasciculations (one dog), absent menace response associated with slow pupillary light reflexes (one dog), injected mucous membranes (two dogs), and abdominal distension (one dog) (Table 1).

\section{Diagnostic tests}

One patient did not have any laboratory tests or diagnostic imaging performed. Complete blood counts and serum biochemistry analyses were performed in the other three dogs, with results outlined in Table 2. Measurements with observed values outside the reference interval (RI) were detected on the complete blood count in two of four dogs (one dog had mature neutrophilia and thrombocytopenia, and one dog had mild reticulocytosis). A manual packed cell volume (PCV) and total plasma protein concentration were determined in two of four dogs: hemoconcentration was evident in two of four dogs, with PCV of $52 \%$ and $67 \%$, respectively. Serum biochemistry measurements were outside the RI in three of four dogs, including decreased inorganic phosphorus concentration (two of four dogs), increased inorganic phosphorus concentration (one of four dogs), increased urea concentration (one of four dogs), increased creatinine concentration (one of four dogs), increased gamma-glutamyl tranferase activity (one of four dogs), increased total bilirubin concentration (one of four dogs), and increased ALT activity. Creatine kinase (CK) activity was only assessed in one dog at presentation, and was moderately elevated (1,815 U/L);

Table 2 Hematology-, biochemistry-, and coagulation-profile results

\begin{tabular}{|c|c|c|c|c|c|}
\hline Analyte & Case I & Case 2 & Case 3 & Case 4 & $\begin{array}{l}\text { Reference } \\
\text { interval }\end{array}$ \\
\hline Urea (mmol/L) & WRI & 29 & Not done & 9.1 & $2.5-9.6$ \\
\hline Creatinine $(\mu \mathrm{mol} / \mathrm{L})$ & WRI & 172 & Not done & 204 & $44-159$ \\
\hline Inorganic phosphorus (mmol/L) & 0.29 & 3.05 & Not done & 0.56 & $0.81-2.2$ \\
\hline Alkaline phosphatase activity (U/L) & WRI & 208 & Not done & 87 & $23-212$ \\
\hline$\gamma$-Glutamyltransferase activity (U/L) & WRI & 13 & Not done & 2 & $0-7$ \\
\hline Total bilirubin $(\mu \mathrm{mol} / \mathrm{L})$ & WRI & 16 & Not done & 8 & $0-15$ \\
\hline Alanine aminotransferase activity (U/L) & WRI & NA & Not done & 65 & $10-100$ \\
\hline Alanine aminotransferase during & WRI & NA & Not done & 775 & $10-100$ \\
\hline \multirow[t]{2}{*}{ hospitalization (U/L) } & & & & 625 & \\
\hline & & & & 58 & \\
\hline Creatine kinase activity (U/L) & NA & NA & Not done & 1,815 & $10-200$ \\
\hline Creatine kinase activity during & NA & NA & Not done & 16,882 & $10-200$ \\
\hline \multirow[t]{3}{*}{ hospitalization (U/L) } & & & & 7,544 & \\
\hline & & & & 1,379 & \\
\hline & & & & 77 & \\
\hline Lactate $(\mathrm{mmol} / \mathrm{L})$ & Not done & Not done & Not done & 2.7 & $<3$ \\
\hline Total plasma protein $(g / L)$ & WRI & 70 & Not done & 73 & $52-82$ \\
\hline Hematocrit (\%) & WRI & 67 & Not done & 55 & $37-61$ \\
\hline Platelet count $\left(\times 10^{9} / \mathrm{L}\right)$ & WRI & 20 & Not done & 264 & $148-484$ \\
\hline aPTT (seconds) & 89 & $>300$ seconds & Not done & 96 & $72-102$ \\
\hline \multirow[t]{6}{*}{ aPTT during hospitalization (seconds) } & $>300,143,109$ & Within reference & Not done & $>300$ & $72-102$ \\
\hline & & interval (number & & $>300$ & \\
\hline & & not specified) & & 204 & \\
\hline & & & & 106 & \\
\hline & & & & 100 & \\
\hline & & & & 110 & \\
\hline PT (seconds) & 19 & $>100$ & Not done & 17 & $11-17$ \\
\hline \multirow[t]{6}{*}{ PT during hospitalization (seconds) } & $>100$ & 19 & Not done & $>100$ & $11-17$ \\
\hline & & & & $>100$ & \\
\hline & & & & $>100$ & \\
\hline & & & & 7 & \\
\hline & & & & 14 & \\
\hline & & & & 17 & \\
\hline
\end{tabular}

Abbreviations: aPTT, activated partial thromboplastin time; NA, not available; PT, prothrombin time; WRI, within reference interval (stated as such, but values not recorded). 
in this patient, alanine transaminase (ALT) activity was within the RI, and aspartate aminotransferase activity was not determined. A repeat CK measurement was made subsequently, with a marked elevation (16,882 U/L) 24 hours after presentation, although seizure activity may have contributed to this increase.

Peripheral oxygen saturation determined using pulse oximetry was reduced $(90 \%$ saturation, RI $>93 \%)$ in the one dog so tested. In-house coagulation measurements were performed in three of four patients at presentation using an in-house Idexx Coag Dx analyzer. Prothrombin time (PT) was prolonged in two of four dogs, with values of 19 seconds and $>100$ seconds (RI 12-17 seconds). Activated partial thromboplastin time (aPTT) was elevated in one of four dogs, with a value of $>300$ seconds. In one patient whose coagulation measurements were normal on presentation, PT and aPTT were prolonged to 100 seconds and $>300$ seconds, respectively, when retested 12 hours after presentation. In another dog whose coagulation times were mildly abnormal at presentation (PT 19 seconds, aPTT within the RI), PT and aPTT were $>100$ seconds and $>300$ seconds, respectively, when retested 12 hours after presentation.

Urinalysis was performed in three of four dogs, and results are summarized in Table 3. Hematuria was present in all cases tested. A snake venom-detection kit (BioSCL Pty Ltd, Melbourne, Australia) was used to assay urine collected within 24 hours of first clinical signs emerging in two of four dogs, both testing negative. In one of those dogs, it was performed twice at 12-hour intervals, both kits testing negative. A urine recreational drug-screening kit (Drug Alert ${ }^{\circledR}$; Frostbland Pty Ltd, Sydney, Australia) capable of detecting amphetamines, barbiturates, benzodiazepines, cocaine, methamphetamine, methylenedioxymethamphetamine, morphine, methadone, opiates, phencyclidine, tricyclic antidepressants, and marijuana was performed in two dogs. Both tested negative.

Table 3 Urinalysis, urine-drug, and venom-screening test results

\begin{tabular}{|c|c|c|c|c|}
\hline Test & Case I & Case 2 & Case 3 & Case 4 \\
\hline $\begin{array}{l}\text { Recreational drug- } \\
\text { detection kit }\end{array}$ & Negative & Not done & Not done & Negative \\
\hline $\begin{array}{l}\text { Snake venom- } \\
\text { detection kit }\end{array}$ & $\begin{array}{l}\text { Negative } \\
\text { on } 2 \text { tests }\end{array}$ & Not done & Not done & Negative \\
\hline Blood (dipstick) & $4+$ & $4+$ & Not done & $2+, 3+$ \\
\hline Bilirubin (dipstick) & Negative & $3+, 2+$ & Not done & Negative \\
\hline pH (dipstick) & 9 & 5,7 & Not done & $6.5,7$ \\
\hline Glucose (dipstick) & Negative & Negative & Not done & Negative \\
\hline Protein (dipstick) & Negative & Negative, trace & Not done & Negative \\
\hline Ketones (dipstick) & Negative & Negative & Not done & Negative \\
\hline Leukocytes (dipstick) & Negative & Negative & Not done & $2+, 3+$ \\
\hline
\end{tabular}

A four-lead electrocardiogram was performed in one patient, demonstrating sinus tachycardia. Thoracic and abdominal imaging (either radiography and/or ultrasound examinations) were performed to screen for the presence of free abdominal fluid in two dogs. No fluid was evident in either case. Previous genetic testing revealed two dogs were polymerase chain reaction-negative for the phosphofructokinase deficiency variant known to occur in ESS, while the other two dogs (cases 1 and 2) were carriers for phosphofructokinase. These two dogs were neither more nor less affected by EIHS signs than the other two dogs.

Case 4 underwent additional genetic testing. Screening for the mutation in the DNM1-gene variant highly associated with exercise-induced collapse in Labrador retrievers was performed by the Veterinary Diagnostic Laboratory, University of Minnesota, and proved to be negative. ${ }^{8}$ A genetic test for the $R Y R I^{\mathrm{V} 547 \mathrm{~A}}$ variant, associated with canine $\mathrm{MH}$ in a study by Roberts et al, was performed by Laboklin in the UK and tested as normal (wild type). ${ }^{9}$

\section{Treatment, outcome, and necropsy findings}

The management of the four cases is summarized in Table 4. Case 1 was treated with aggressive intravenous (IV) crystalloids (Hartmann's solution), active cooling by hosing limbs using cold tap water, oxygen supplementation via a tightfitting face mask, IV metronidazole, and sedation using IV acepromazine and IV butorphanol. The dog developed diarrhea, without hematochezia or melena. Hyperthermia resolved within 30 minutes of initiating treatment. Within 2 hours, the patient was ambulatory and had an improved demeanor. The dog subsequently developed a coagulopathy within 12 hours of presentation. Ongoing therapy included IV ticarcillin clavulanate, subcutaneous (SC) maropitant, four transfusions of fresh frozen plasma, IV esomeprazole and oral vitamin $\mathrm{K}_{1}$ supplementation. He recovered fully, and was discharged 72 hours after presentation.

Case 2 was treated with vitamin K, IV crystalloids, a single injection of dexamethasone sodium phosphate, IV cefazolin, and IV enrofloxacin at the primary veterinary clinic and then referred to a veterinary specialist center. Vitamin $\mathrm{K}_{1}$ supplementation was continued, together with a unit of frozen plasma, IV maropitant, and IV ampicillin. The patient developed profuse salivation and diarrhea with melena. He made a full recovery, and was discharged 48 hours after presentation.

Case 3 developed vomiting, diarrhea, hematochezia, and melena. He was treated with IV crystalloids (Hartmann's 
Table 4 Treatment administered within first 48 hours of admission

\begin{tabular}{|c|c|c|c|c|}
\hline Treatment & Case I & Case 2 & Case 3 & Case 4 \\
\hline Methadone & No & Unknown & $0.2 \mathrm{mg} / \mathrm{kg} \mathrm{IM}$ & $0.1 \mathrm{mg} / \mathrm{kg}$ IV PRN \\
\hline Butorphanol & $0.2 \mathrm{mg} / \mathrm{kg}$ IV & No & No & No \\
\hline Active cooling & Hosing of pelvic limbs & No & No & No \\
\hline $\begin{array}{l}\text { Oxygen } \\
\text { supplementation }\end{array}$ & Via face mask & No & No & No \\
\hline IV fluids & $\begin{array}{l}\text { Hartmann's solution at } \\
140 \mathrm{~mL} / \mathrm{h}\end{array}$ & $\begin{array}{l}\text { Hartmann's, unknown } \\
\text { rate }\end{array}$ & $\begin{array}{l}\text { Yes, unrecorded type } \\
\text { and rate }\end{array}$ & Hartmann's at $125 \mathrm{~mL} / \mathrm{h}$ \\
\hline Antimicrobials & $\begin{array}{l}\text { Metronidazole, unknown dose } \\
\text { IV every } 12 \text { hours; ticarcillin- } \\
\text { clavulanic acid } 50 \mathrm{mg} / \mathrm{kg} \text { rate } \\
\text { IV every } 8 \text { hours }\end{array}$ & $\begin{array}{l}\text { Cefazolin, unrecorded } \\
\text { dose IV; enrofloxacin } \\
5 \mathrm{mg} / \mathrm{kg} \text { IV }\end{array}$ & $\begin{array}{l}\text { Metronidazole, unknown } \\
\text { dose IV; amoxicillin- } \\
\text { clavulanic acid unknown } \\
\text { dose SC }\end{array}$ & $\begin{array}{l}\text { Metronidazole } 10 \mathrm{mg} / \mathrm{kg} \text { IV every } \\
12 \text { hours; ampicillin } 20 \mathrm{mg} / \mathrm{kg} \text { IV } \\
\text { every } 8 \text { hours }\end{array}$ \\
\hline Vitamin $\mathrm{K}$ & $2.5 \mathrm{mg} / \mathrm{kg} \mathrm{PO}$ & $\begin{array}{l}\text { Yes, but dose and } \\
\text { route of administration } \\
\text { unrecorded }\end{array}$ & No & $2.5 \mathrm{mg} / \mathrm{kg} \mathrm{SC}$ \\
\hline $\begin{array}{l}\text { Fresh frozen plasma } \\
\text { transfusion }\end{array}$ & 4 units & No & No & 2 units \\
\hline Other & $\begin{array}{l}\text { Maropitant I mg/kg SC every } \\
24 \text { hours; esomeprazole } 0.7 \\
\mathrm{mg} / \mathrm{kg} \text { IV every } 24 \text { hours }\end{array}$ & $\begin{array}{l}\text { Dexamethasone } 0.25 \mathrm{mg} / \\
\mathrm{kg} \mathrm{SC}\end{array}$ & NA & $\begin{array}{l}\text { Levetiracetam I } 6 \mathrm{mg} / \mathrm{kg} \text { IV every } \\
8 \text { hours; esomeprazole I mg/kg IV } \\
\text { every } 24 \text { hours; maropitant I mg/kg } \\
\text { IV every } 24 \text { hours; metoclopramide } \\
\text { I mg/kg/day IV; sucralfate I g PO } \\
\text { every I } 2 \text { hours }\end{array}$ \\
\hline
\end{tabular}

Abbreviations: IM, intramuscular; IV, intravenous; NA, not applicable; PO, per os (by mouth); PRN, pro re nata (as needed); SC, subcutaneous.

solution), SC amoxicillin-clavulanic acid, IV metronidazole, and intramuscular (IM) methadone. The following day, the patient remained recumbent, but was responsive. He continued to have hemorrhagic diarrhea and developed petechiation, although further laboratory assessment of hemostasis was not undertaken. He suffered a cardiac arrest, and resuscitation attempts were unsuccessful. Necropsy examination and histological examination of tissues collected postmortem showed accelerated autolytic changes and mild neutrophilic mucosal infiltration of the small bowel, plus loss of mucosal epithelium associated with severe acute hemorrhage into the intestinal lumen. There was also severe acute hemorrhage in multiple other organs, particularly the lungs, stomach, and liver. There was no definitive histological evidence for a specific causation, and no evidence of a preexisting pathological process that might have accounted for this etiopathogenesis. Thrombosis of large or small vessels, consistent with disseminated intravascular coagulation (DIC), was not apparent.

Case 4 was treated with IV crystalloids, $\mathrm{SC}$ vitamin $\mathrm{K}_{1}$, IV maropitant, IV metronidazole, IV ampicillin, IV esomeprazole, IV levetiracetam, oral sucralfate, and 2 units of fresh frozen plasma. He developed hematochezia, melena, diarrhea, and vomiting with hematemesis. He was discharged 3 days after presentation, with complete resolution of all clinical signs.

\section{Familial occurrence}

Pedigree information was available for all four dogs and eleven related dogs (Figure 1A). The genetic relationship between the four affected dogs and 37 additional related individuals is presented in Figure 1. Two dogs were siblings. All four dogs were closely related, with a common grandsire from generation I (Figure 1A). Additional investigation beyond additional pedigree analysis into affected and unaffected littermates and related dogs was recommended but declined. There is no known history of any other related dogs or related dogs being affected by this type of clinical scenario. From pedigree observation, recessive sex-linked genetic transmission was hypothesized (Figure 1B), although an autosomal-recessive mode of inheritance cannot be excluded. Blood (ethylenediaminetetraacetic acid [EDTA]-anticoagulated and frozen) from the surviving dogs and tissue blocks from the dog that died have been archived for planned genetic investigations.

\section{Discussion}

The objective of this study was to further define EIHS/CSS in a kindred of ESS dogs and to attempt to determine if there was an identifiable mechanism for genetic inheritance. To summarize the clinical findings, all dogs developed broadly similar signs in response to mild-to-moderate exercise. The dogs were housed separately and developed EIHS/CSS under different specific circumstances, suggesting that the 
A

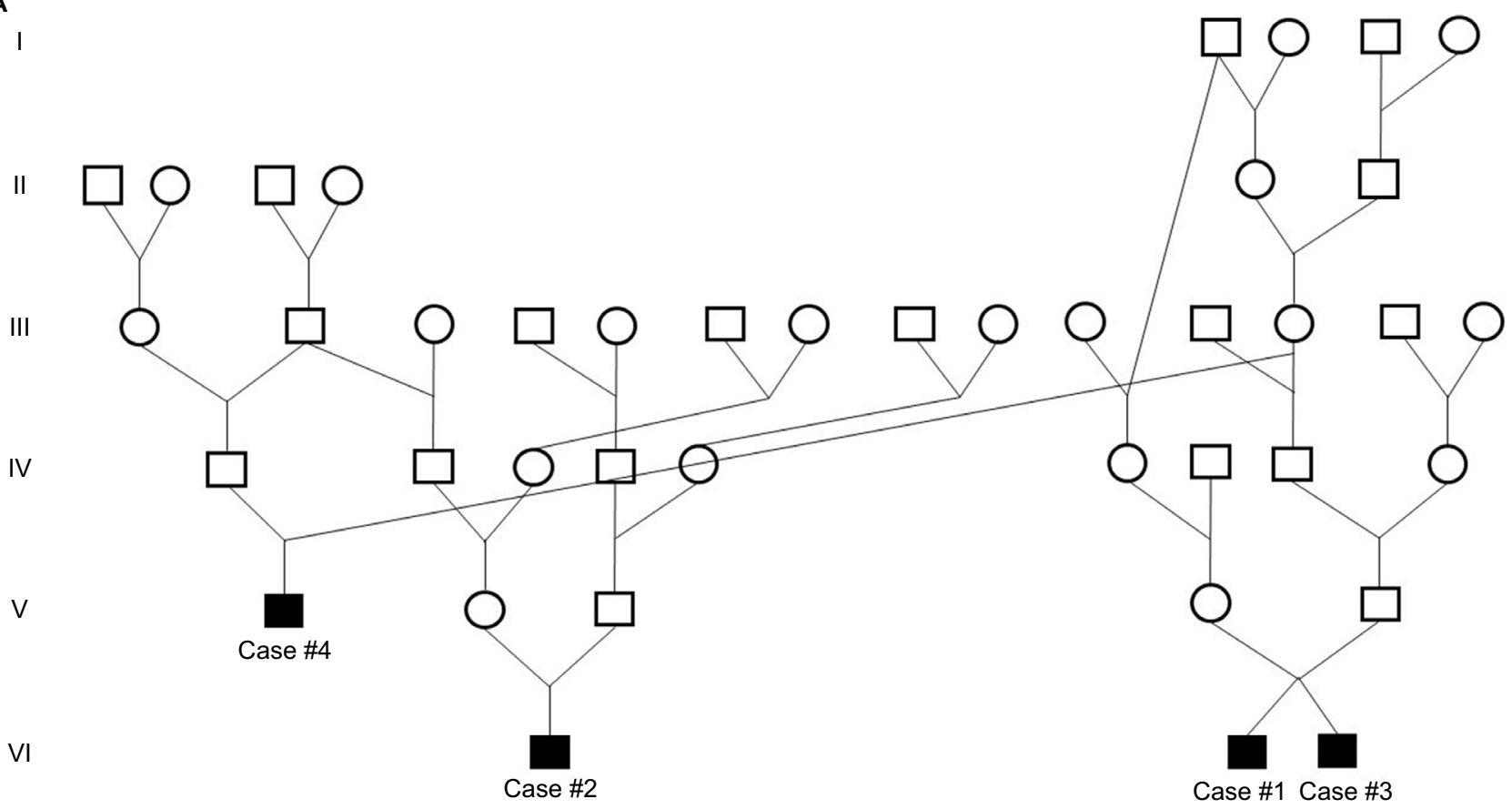

B

I

II

III
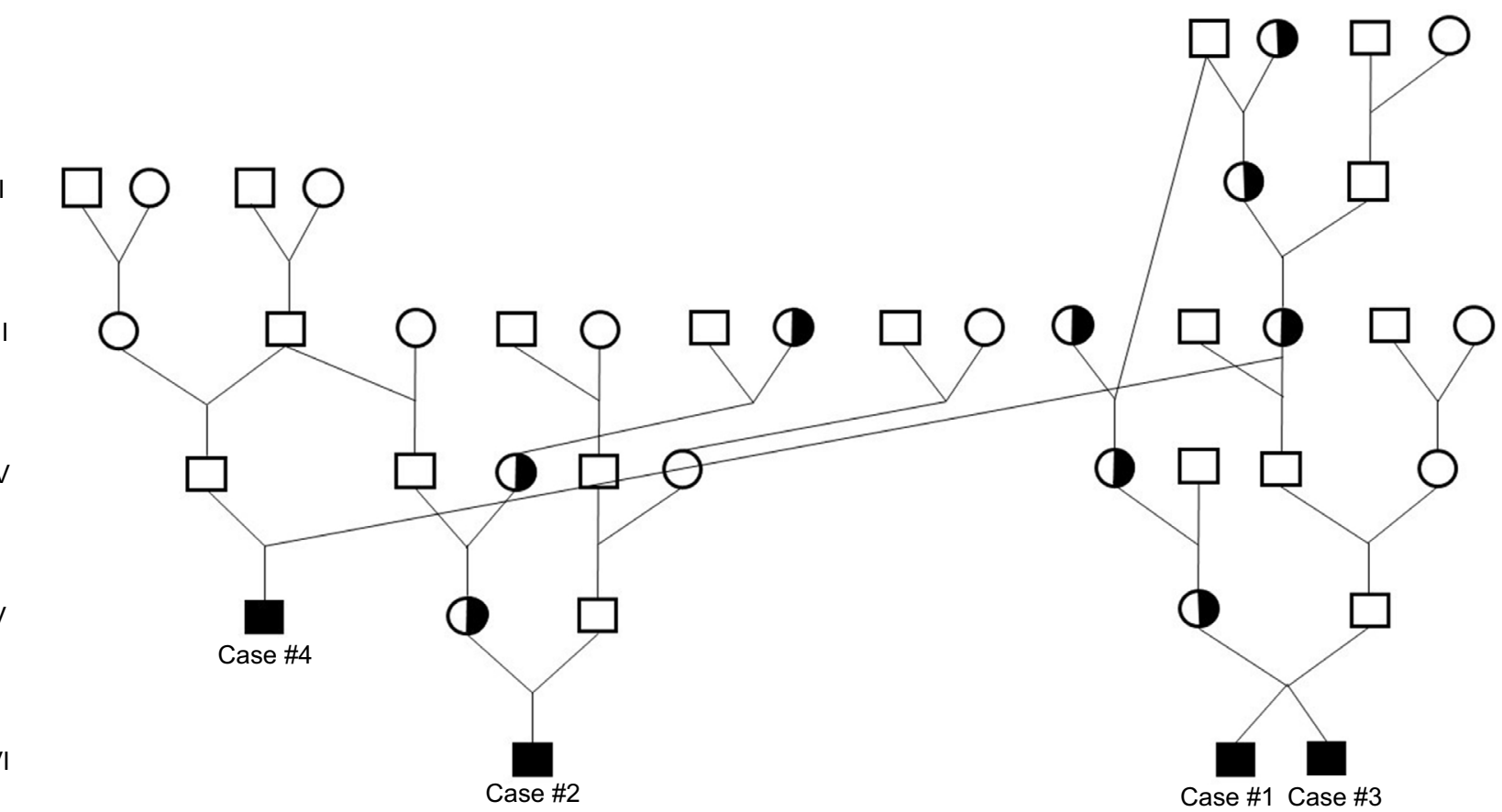

Figure I Multigenerational pedigree representing affected males segregating for canine stress syndrome.

Notes: Circles represent female and squares represent males. Solid symbols represent the affected dogs included in the study. (A) Pedigree representing the relationship between the four affected males and 37 additional related individuals. (B) Pedigree hypothesizing the inheritance of the disease allele under a recessive X-linked transmission. Half-filled circles represent the hypothesized inheritance of mutated allele from the maternal side.

syndrome was not related to some common environmental circumstance or toxicant. Apart from moderate exertion, no other triggers were identified. The dogs were closely related intact males, including two littermates. This raises the possibility that the predisposition to the development of this syndrome is inherited more likely as a recessive sex-linked trait or less likely as an autosomal-recessive condition, as the chance of an autosomal trait affecting four of four male 
dogs would be $6 \%$. If consideration is made of the previous ESS reported from Canada, also of male sex, then the likelihood of sex-linked inheritance is even higher. ${ }^{6}$ Analysis of the genetic relationship between the four affected dogs from Australia for which we have a pedigree is consistent with sex-linked inheritance, although at this stage in our investigations autosomal-recessive transmission cannot be completely excluded (Figure 1). Further observations and studies should be able to establish whether all putative carrier females have a common female ancestor, consistent with sex-linked inheritance. The presence of four cases from Australia and one from Canada suggested the causal gene is likely to be well dispersed among the ESS population worldwide.

In human medicine, the variability of presentation of $\mathrm{MH}$ has resulted in it being considered a syndrome, rather than a single disease. ${ }^{3}$ This syndrome results from the breakdown of the calcium-sequestration mechanism in the sarcoplasmic reticulum of skeletal muscle. Multiple genetic and environmental factors affect the way the disease is expressed phenotypically. ${ }^{2,3}$ In man, susceptibility to $\mathrm{MH}$ is often inherited as an autosomal-dominant disorder, for which there are at least six genetic loci of interest, most prominently $R Y R 1 .{ }^{10,11} \mathrm{MH}$ susceptibility is also phenotypically and genetically related to central core disease, an autosomal-dominant disorder characterized both by $\mathrm{MH}$ signs and myopathy. Most people with MH susceptibility have few or no symptoms or signs unless they are exposed to a triggering agent, and the same is true of the equivalent disease in pigs. ${ }^{1,12}$ The most common triggering agents are volatile anesthetic gases or depolarizing muscle relaxants. In rare cases, the biological stresses of physical exercise or heat may act as the trigger. ${ }^{1,2,13}$

There is a limited veterinary literature suggesting that abnormal calcium release from the sarcoplasmic reticulum of skeletal muscle can be triggered by exercise or stress in dogs with a particular genetic background, just as multiple triggers for the syndrome's expression exist in the pig. ${ }^{5}$ This EIH or CSS has been reported previously in an ESS and a greyhound, and there are anecdotal reports of further cases. ${ }^{6,7}$

The seminal report of exercise-induced $\mathrm{MH}$ in an ESS was described by Rand and O'Brien. ${ }^{6}$ The dog was 2 years of age and male, comparable to the dogs of the present study. In this report, there was increased erythrocyte osmotic fragility, hypercontracted muscle fibers, and muscle fasciculations after inhalation of halothane, consistent with $\mathrm{MH}$. The dog also demonstrated muscular hypersensitivity to caffeine, a more definitive diagnostic in vitro test for $\mathrm{MH}$. However, prophylactic administration of dantrolene (a drug that specifically blocks $\mathrm{Ca}^{2+}$ release from the sarcoplasmic reticulum) did not prevent EIH in this individual. The authors referred to this presentation as "aborted malignant hyperthermia reaction", a "malignant hyperthermia-like reversible syndrome", or "canine stress syndrome", and it seems likely that whatever the condition is called, the four dogs we report here were afflicted by the same entity.

Three of the cases described occurred independently, without awareness of similar clinical signs in other affected dogs. The final case (case 4) presented to the primary author after she had the opportunity of reviewing the case notes of the three previous cases. Based on this characteristic "illness script" or anchoring heuristic (ie, true anchor), EIHS/CSS was considered the most likely diagnostic possibility. ${ }^{14-16}$ This logical shortcut facilitated the rapid selection of appropriate diagnostic tests and a cogent targeted treatment regimen. Unfortunately, additional investigations specific to $\mathrm{MH}$ were not undertaken, as the caffeine contracture test is invasive and thus expensive (as it requires collection of a muscle biopsy under general anesthesia, plus the costs of testing at a specialist human laboratory), and the halothane-challenge test was considered an unacceptable risk to the patient, especially as IV dantrolene was not readily available. It would be ideal, however, if such tests were undertaken, and supplemented by the full range of histochemical tests on fresh frozen muscle biopsies at a specialist laboratory, possibly including transmission electron microscopy.

In all four ESS dogs, it was thought that the clinical changes induced by exercise were considerably disproportionate to the intensity of the effort, even when factoring in additional elements, such as environmental temperature, humidity, and the like. As a result, our speculation was that these dogs likely: 1) had an underlying myopathy with the potential to cause EIH, yet not sufficiently severe to meet the stringent criteria for classic $\mathrm{MH}$; that 2) hyperthermia and or changes in skeletal muscle somehow gave rise to secondary disease processes, in which a DIC-like consumptive coagulopathy was prominent; and 3 ) this in turn resulted in gastrointestinal ischemia, diarrhea with or without blood loss into the gut lumen, and seizures. The delayed elevation of CK activity in one patient (case 4) provides supporting evidence for a primary myopathic process, possibly with a component of reperfusion injury (consistent with the observed delay in increased CK activity in serum), with a secondary cascade of changes (perhaps triggered by hyperthermia or key molecules released from affected myofibers) impacting on numerous body systems. However, CK elevation can be associated with muscle damage, and may not be associated with primary muscle disease. Therefore, the cause of the increase in CK from $1,815 \mathrm{U} / \mathrm{L}$ before the seizure to $16,882 \mathrm{U} / \mathrm{L}$ after the seizure may have been multifactorial. A weakness of the 
present case studies was absence of serial determinations of $\mathrm{CK}$ activity and urinary myoglobin, initially at presentation and subsequently. CK activity was not determined in the remaining dogs, as they were seen as emergencies, and this enzyme is not typically included in the cassettes and panels used for routine in-house biochemistry testing in the respective emergency facilities. Delayed disease features, such as coagulopathy and consumptive thrombocytopenia, provide circumstantial evidence for such a pathophysiologic chronology. The reason for ancillary testing for snake envenomation in many cases with coagulopathy reflects the importance of this entity in Australia (ie, availability heuristic), while the commonness of anticoagulant rodenticide poisoning explains the frequency with which vitamin $\mathrm{K}_{1}$ was administered "just in case". ${ }^{14}$ The reason for hematuria perhaps reflected the generalized tendency to bleed and a leaky glomerular barrier.

There may be some similarities with exercise-induced rhabdomyolysis syndromes, which have limited representation in the veterinary literature, but are more widely reported in human and horses. Hematuria, however, was documented in case 4, rather than myoglobinuria, and what is important in the dogs investigated in this study is the exercise was not considered to be extreme or excessive either immediately prior to or in the 24 hours preceding the development of signs. All four dogs were considered fit, and exercised regularly. Most of the exercise-induced inherited metabolic myopathies in people result in rhabdomyolysis and myoglobinuria. ${ }^{15}$ However, in people with $\mathrm{MH}$, myoglobinuria typically occurs only in the most severe cases. ${ }^{15}$

The University of Cambridge Veterinary School's Inherited Diseases in Dogs Database lists MH in ESSs as being of unknown inheritance. It is pertinent that all four cases of the present report and the earlier case from Canada occurred in ESS dogs. Furthermore, the four affected dogs were all male. All four dogs had a common grandsire, which would be spurious if the inheritance was indeed sex-linked, as of the sex chromosomes, only the $\mathrm{Y}$ chromosome is transmitted from sires to male offspring. Given the close familial relationship of these breeds, provocative testing of putative female carriers as well as affected dogs would seem compelling, if possible. Obtaining DNA specimens (via EDTA blood or cheek swabs) from all affected and closely related dogs would seem logical, with a view to either: 1) sample as many individuals from the pedigree as possible and utilize the canine single-nucleotide polymorphism chip to perform a genome-wide association scan to localize the trait to a chromosomal region (perhaps on the X chromosome) and scan for candidate genes or 2) obtain whole-genome sequences for a "trio" consisting of one affected male dog, an unaffected female sibling (such as an older sibling known to have been subjected to heavy exercise and/or thermal stress without developing any signs), and one parent (dam or sire).

The test for the RYRl-gene variant associated with canine $\mathrm{MH}$ was negative in case 4 . This specific mutation was identified by Roberts et al, and results in autosomaldominant canine $\mathrm{MH}$, the gene encoding the skeletal muscle calcium-release channel (RYRl). ${ }^{9}$ Historically, researchers interested in the molecular basis for canine $\mathrm{MH}$ established a breeding colony starting with a mixed-breed male dog that had survived an in vivo halothane-succinylcholine challenge. However, one of the contributing authors of the aforementioned paper from the Department of Veterinary Biomedical Sciences, University of Minnesota indicated the search for that specific canine variant had stopped, since they never found another dog with that mutation in over 24 subsequent tests (J Mickelson, personal communication).

Case 4 was negative when tested for the DMN1-gene variant associated with exercise-induced collapse in Labradors. ${ }^{8}$ In this syndrome, dogs develop an abnormal gait, tachycardia, and significant respiratory alkalosis after exercise. However, cases of EIC have not yet been described as having other systemic signs, such as coagulopathy.

Future investigation of the EIHS/CSS phenotype would include detailed assessment of well-characterized canine patients, serial determination of CK activity (and also ALT and aspartate aminotransferase activities) during an attack, and also (after vigorous exercise or thermal stress) conducting electrodiagnostic studies under general anesthesia in an attempt to detect a cryptic myopathy, obtaining fresh and fixed muscle-biopsy specimens for detailed histological and ultrastructural investigations, again in the search of a myopathy predisposing to $\mathrm{MH}$ or an $\mathrm{MH}$-like episode, and archiving DNA from all affected dogs, their normal siblings, and parents, to permit future molecular investigations harnessing the power of modern canine genomics. In a study by O'Brien et al, erythrocyte osmotic fragility and CK tests did not distinguish between what they termed a "malignanthyperthermia" dog and healthy control dogs. ${ }^{4}$

The lack of muscle biopsies in these cases reflects the limitation of a retrospective study. Postexercise lactate and pyruvate levels were not determined, and such measurements may have been useful in characterizing the cause if myopathy was the primary underlying condition. However, a serum lactate determination was in the RI in case 4 within 24 hours of presentation.

None of the four cases had a measured core body temperature that one would have expected to cause DIC or any other of the sequelae associated with heatstroke. Normal 
dogs can have a body temperature greater than $41^{\circ} \mathrm{C}$ with strenuous exercise.${ }^{17}$ However, rectal temperature at the time of exercise intolerance was never recorded, and all cases had some delay prior to seeking veterinary attention.

Once the genetic variant is characterized, a simple polymerase chain reaction test can be developed and used to screen the entire ESS breed worldwide and exclude carriers from the breeding program. This should enable the detection of both carriers and dogs at risk for EIHS/CSS. In addition, it might represent an interesting model for some corresponding human disease state. Ultimately, the greatest value in developing such a test is for detecting dogs that might potentially be subjected to stressful triggers, which might then give rise to a life-threatening crisis. Identified dogs can then be appropriately monitored.

Despite one of the four dogs dying in this study, dogs suffering from EIHS/CSS can make a speedy recovery if given aggressive supportive therapy (often with the requirement for fresh frozen plasma). Indeed, the severity of the disease and rapid turnaround with timely supportive therapy appear to be distinguishing features of this entity. Awareness of the features of this syndrome that develop after presentation, such as coagulopathy, signaling the requirement for fresh frozen plasma or even fresh whole blood, will likely improve survival of affected dogs.

\section{Conclusion}

This study analyzed the potential for inheritance of suspected CSS in the ESS breed, due to the similar presentation of four young male ESSs, as well as describing the clinical syndrome of CSS, with discussion of the pertinent yet limited veterinary literature concerning CSS. Common causes of exercise intolerance were eliminated in the cases included in the study, but the exact cause is yet to be determined. This study provides evidence that the mode of inheritance is more likely X-linked and unlikely (although possible) to be autosomal-recessive. Further genetic characterization and testing should be strongly considered by those involved with the ESS breed. Identification of this syndrome may allow for closer observation of related dogs that may have a genetic predisposition for this disease but remain asymptomatic, as well as identifying any possible triggers that precipitate clinical signs.

\section{Acknowledgment}

The authors would like to thank Connor Pyne for his help with the pedigree preparation.

\section{Disclosure}

The authors report no conflicts of interest in this work.

\section{References}

1. Soma P, van Marle-Köster E, Frylinck L. Frequency of the malignant hyperthermia gene in the South African pig industry. SAfr J Anim Sci. 2015;44(4):384-387.

2. Rosenberg H, Davis M, James D, Pollock N, Stowell K. Malignant hyperthermia. Orphan J Rare Dis. 2007;2(1):21.

3. Brunson DB, Hogan KJ. Malignant hyperthermia: a syndrome not a disease. Vet Clin North Am Small Anim Pract. 2004;34(6):1419-1433.

4. O'Brien PJ, Pook HA, Klip A, et al. Canine stress syndrome/malignant hyperthermia susceptibility: calcium homeostasis defect in muscle and lymphocytes. Res Vet Sci. 1990;48(1):124-128.

5. Le Couteur RA. Neuropathies, junctionopathies and myopathies of dogs and cats. Poster presented at: Second Annual Veterinary Neurology Symposium; July 23-24, 2005. Davis, CA.

6. Rand JS, O'Brien PJ. Exercise-induced malignant hyperthermia in an English springer spaniel. J Am Vet Med Assoc. 1987;190(8):1013-1014.

7. Dickinson PJ, Sullivan M. Exercise induced hyperthermia in a racing greyhound. Vet Rec. 1994;135(21):508.

8. Patterson EE, Minor KM, Tchernatynskaia AV, et al. A canine dynamin 1 mutation is highly associated with the syndrome of exercise-induced collapse. Nat Genet. 2008;40(10):1235-1239.

9. Roberts MC, Mickelson JR, Patterson EE, et al. Autosomal dominant canine malignant hyperthermia is caused by a mutation in the gene encoding the skeletal muscle calcium release channel (RYR1). Anesthesiology. 2001;95(3):716-725.

10. Litman R, Rosenberg H. Malignant hyperthermia: update on susceptibility testing. JAMA. 2005;293(23):2918-2924.

11. Groom L, Muldoon SM, Tang ZZ, et al. Identical de novo mutation in the type 1 ryanodine receptor gene associated with fatal, stress-induced malignant hyperthermia in two unrelated families. Anesthesiology. 2011;115(5):938-945.

12. Schneiderbanger D, Johannsen S, Roewer N, Schuster F. Management of malignant hyperthermia: diagnosis and treatment. Ther Clin Risk Manag. 2014;10:355-362.

13. Litman R, Flood CD, Kaplan RF, Kim YL, Tobin JR. Postoperative malignant hyperthermia: an analysis of cases from the North American Malignant Hyperthermia Registry. Anesthesiology. 2008; 109(5):825-829

14. Canfield PJ, Whitehead ML, Johnson R, O'Brien CR, Malik R. Case-based clinical reasoning in feline medicine 1: intuitive and analytical systems. J Feline Med Surg. 2015;18(1):35-45.

15. Shelton GD. Rhabdomyolysis, myoglobinuria, and necrotizing myopathies. Vet Clin North Am Small Anim Pract. 2004;34(6): 1469-1482.

16. Whitehead ML, Canfield PJ, Johnson R, O’Brien CR, Malik R. Casebased clinical reasoning in feline medicine 3: use of heuristics and illness scripts. J Feline Med Surg. 2016;18(5):418-426.

17. Steiss JE, Wright JC. Respiratory alkalosis and primary hypocapnia in Labrador retrievers participating in field trials in high-ambienttemperature conditions. Am J Vet Res. 2008;69(10):1262-1267. 
Veterinary Medicine: Research and Reports is an international, peer-reviewed, open access journal publishing original research, case reports, editorials, reviews and commentaries on all areas of veterinary medicine. The manuscript management system is completely online and includes a very quick and fair peer-review system.
Visit http://www.dovepress.com/testimonials.php to read real quotes from published authors.

Submit your manuscript here: http://www.dovepress.com/veterinary-medicine-research-and-reports-journal 